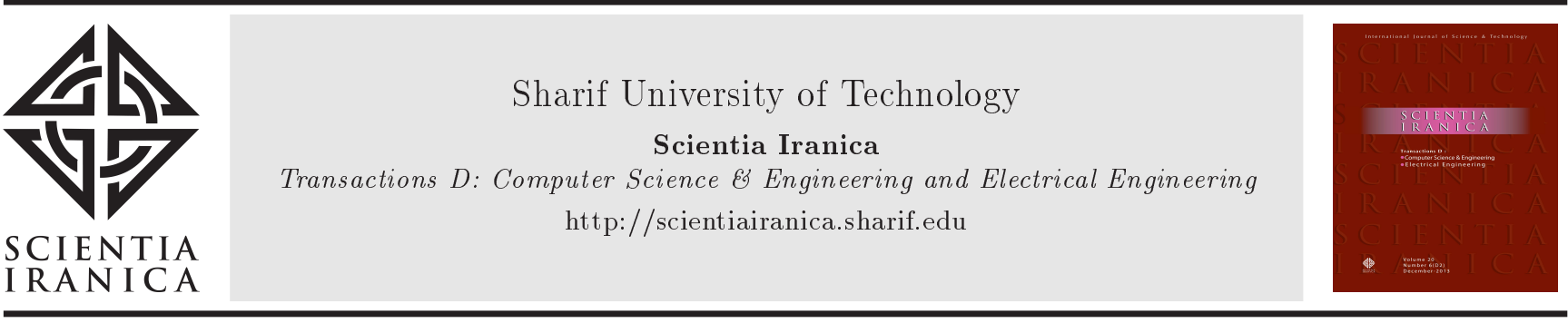

Research Note

\title{
Tunable active grounded lossless and lossy inductance simulators with single grounded capacitor using VDBAs
}

\author{
W. Tangsrirat ${ }^{\mathrm{a}, *}$ and W. Surakampontorn ${ }^{\mathrm{b}, \mathrm{c}}$ \\ a. School of Engineering, King Mongkut's Institute of Technology Ladkrabang (KMITL), Chalongkrung Road, Ladkrabang, Bangkok \\ 10520, Thailand. \\ b. College of Advanced Manufacturing Innovation, King Mongkut's Institute of Technology Ladkrabang (KMITL), Chalongkrung \\ Road, Ladkrabang, Bangkok 10520, Thailand. \\ c. Academy of Science, Royal Society of Thailand, Dusit Palace Ground, Dusit, Bangkok 10300, Thailand.
}

Received 20 August 2018; received in revised form 16 August 2019; accepted 23 December 2019

\author{
KEYWORDS \\ Voltage Differencing \\ Buffered Amplifier \\ (VDBA); \\ Lossless inductor; \\ Lossy inductor; \\ Grounded inductance \\ simulator; \\ Electronically tunable \\ circuit.
}

\begin{abstract}
This study presents three active-C synthetic grounded inductance simulator circuits that realize tunable lossless and lossy series and parallel R-L type inductances. Each of these circuits employs two Voltage Differencing Buffered Amplifiers (VDBAs) as active components and a single grounded capacitor as a passive component. In all the proposed circuits, the simulated equivalent resistance and inductance values can be adjusted electronically through the transconductance gains of the VDBAs. They also do not require any critical component matching conditions and cancellation constraints. Details of non-ideal analysis including transfer errors of the VDBA has been analyzed. For circuit performance verification and comparison, some application examples are given together with computer simulation results by PSPICE program.
\end{abstract}

(C) 2022 Sharif University of Technology. All rights reserved.

\section{Introduction}

Inductors are one of the most essential circuit components of analog and mixed-signal integrated circuits and systems. Particularly, they are used to realize a variety of active networks, such as active ladder filters, impedance matching circuitry, and chaotic oscillators [1]. However, in the modern design of integrated circuits, the integration of high-valued inductors is a fundamental problem because of the occupied silicon area. In addition, their values cannot adjust easily after fabrication. Owing to these restrictions, numerous

\footnotetext{
*. Corresponding author. Tel./Fax: +662-326-4205 E-mail addresses: worapong.ta@kmitl.ac.th (W. Tangsrirat); wanlop.ltpw@gmail.com (W. Surakampontorn)
}

doi: $10.24200 /$ sci. 2019.51551 .2248 synthetic simulated inductors using different kinds of active elements have been created instead of largevalued physical inductors [2-16]. These simulators can be classified based on whether they realize a grounded or floating inductor configuration or whether they simulate a lossless or lossy type of inductor. In the class of grounded inductance simulators, they have a simpler structure than floating ones. Further, from these simulators, some of them are related to simulation of grounded lossless inductor [2-8]. Moreover, several specific configurations for simulating series and parallel R-L type lossy inductor are also available in [916]. Among these simulators, the circuits in $[4,6,10]$ require two active devices and three or more passive elements for simulating grounded lossless and parallel R-L-type lossy inductors. In the realization in [2$3,5,7,9,11-15]$, only one active device and at least three passive elements are employed to simulate grounded 
inductances. In addition to the circuits of $[2-4,6-$ 16], they are realized with ungrounded resistors, which are impediments in Integrated Circuit (IC) fabrication. Most of them also include a floating capacitor [2-3,6$8,10-14]$. It is well known that the use of only grounded capacitors particularly makes the circuits suitable for fully IC design [17]. Although the lossless grounded inductor realizations discussed in $[8,16]$ employ only one active component, they still contain two floating passive components for their realizations. Moreover, the synthetic inductors developed in $[2-7,9-14]$ do not possess the property of electronic tunable performance. Some of the above-mentioned simulators also suffer from the need for component-matching constraints $[2-3,5-7,12]$, where some capacitors or resistors are needed to either satisfy certain conditions and/or have identical values. In the literature, the external adjustability of circuits without requirement for any critical component-matching choices has considerable advantages for IC design [18-19].

Recently, the Voltage Differencing Buffered Amplifier (VDBA), which is a novel introduced versatile active building block, has been introduced and also applied to design and synthesis of diverse analog circuit functions and applications [20-21]. The VDBA essentially combines the electronic tuning feature of the classical transconductance amplifier together with the voltage following property of the output voltage buffer. In comparison to the existing active elements, the VDBA-based circuit does not need any additional resistors for voltage-to-current conversion in some voltagemode application circuits similar to the case of the current differencing buffered amplifier-based circuit [22]. Compared to the existing transconductance-based active devices such as Current Differencing Transconductance Amplifier (CDTA), Current Conveyor Transconductance Amplifier (CCTA), and Differential Voltage CCTA (DV-CCTA), the VDBA device allows lowimpedance voltage output, which is directly cascadable in voltage-mode operations owing to the effect of load capacitance or resistance changing can be avoided [20].

To demonstrate the utility and viability of the VDBA device, three canonical grounded inductance simulator circuits with a single grounded capacitor are proposed. Out of the three proposed circuits, the first circuit realizes a lossless or pure inductor, while two circuits realize series and parallel lossy inductors. In all the proposed inductor realizations, only two VDBAs and one grounded capacitor are employed. The simulated equivalent resistance and inductance values can be tuned electronically by adjusting the bias currents of the VDBAs. Computer simulations based on TSMC $0.25-\mu \mathrm{m}$ CMOS real process parameters are performed to validate the circuit operation. In addition to the utility of the proposed simulator circuits, some illustrative applications are also given.

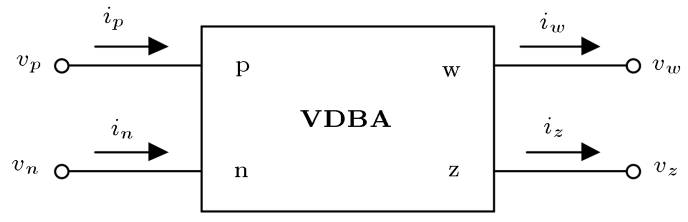

Figure 1. Symbolic representation of the VDBA.

\section{Voltage Differencing Buffered Amplifier (VDBA)}

As a symbolic notation represented in Figure 1, the VDBA is an active building block with four terminals, namely $\mathrm{p}, \mathrm{n}, \mathrm{z}$, and $\mathrm{w}$ [20-21]. The defining operation of the VDBA can be expressed by the following equation:

$$
\left[\begin{array}{c}
i_{p} \\
i_{n} \\
i_{z} \\
v_{w}
\end{array}\right]=\left[\begin{array}{cccc}
0 & 0 & 0 & 0 \\
0 & 0 & 0 & 0 \\
\alpha g_{m} & -\alpha g_{m} & 0 & 0 \\
0 & 0 & \beta & 0
\end{array}\right] \cdot\left[\begin{array}{c}
v_{p} \\
v_{n} \\
v_{z} \\
i_{w}
\end{array}\right]
$$

where $g_{m}$ is the transconductance value; and $\alpha$ and $\beta$ are the non-ideal transfer gains of the VDBA, respectively. Note that the values of $\alpha$ and $\beta$ are equal to unity in the ideal case.

\section{Proposed inductance simulators}

The proposed circuits for simulating the grounded lossless and lossy inductors are shown in Figures 24. It can be observed that each of the proposed circuits consists of solely two VDBAs and a single grounded capacitor without the requirement of any external resistors. Under ideal conditions $(\alpha \cong \beta \cong 1)$, straightforward analysis of the circuits in Figures 24 yields their input impedances to be expressed as follows:

For the circuit of Figure 2:

$$
Z_{i n 1}=\frac{s C_{L}}{g_{m 1} g_{m 2}}=s L_{e q 1},
$$

For the circuit of Figure 3:

$$
Z_{i n 2}=\frac{1}{g_{m 1}}+\frac{s C_{S}}{g_{m 1} g_{m 2}}=R_{e q 2}+s L_{e q 2},
$$

For the circuit of Figure 4:

$$
Z_{i n 3}=\frac{s C_{P}}{g_{m 1} g_{m 2}+s C_{P} g_{m 1}}=R_{e q 3} / / s L_{e q 3},
$$

where $g_{m i}(i=1,2)$ stands for the parameter $g_{m}$ associated with the $i$ th VDBA. Therefore, the proposed circuit of Figure 2 simulates the lossless inductor, while the circuits of Figures 3 and 4 realize the series and 


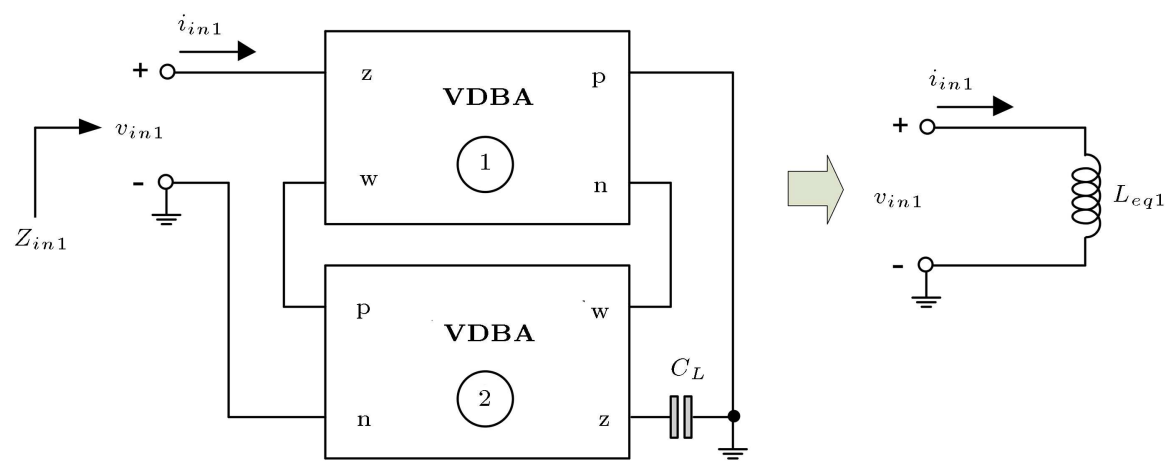

Figure 2. Proposed grounded lossless inductance simulator.

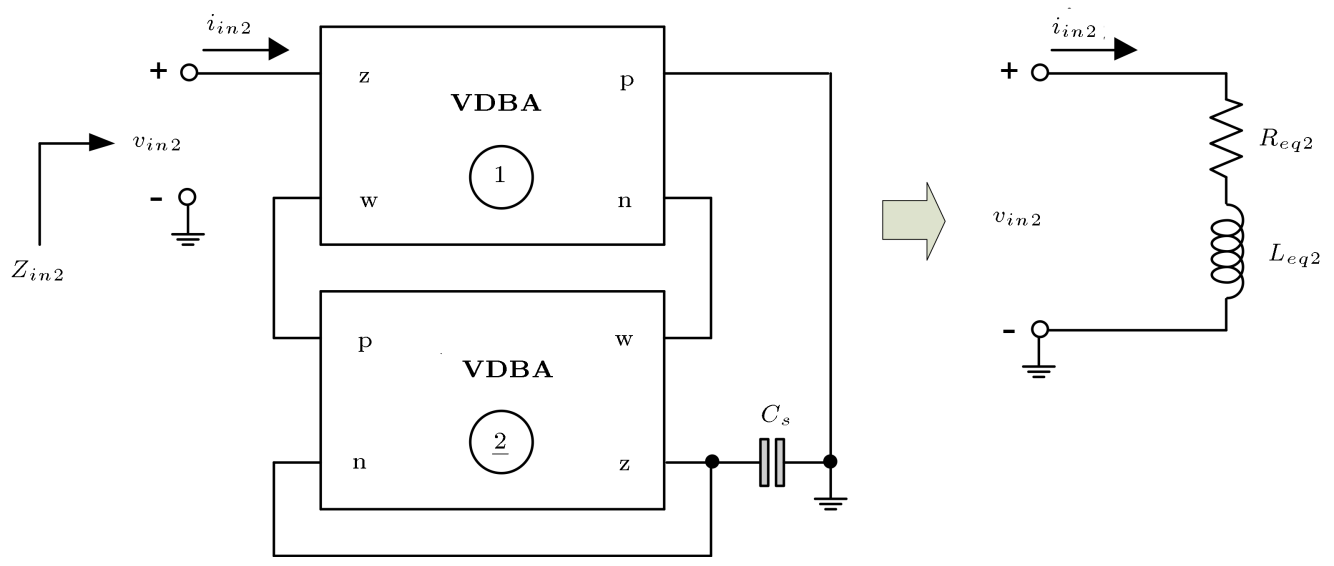

Figure 3. Proposed grounded series-R-L type lossy inductance simulator.

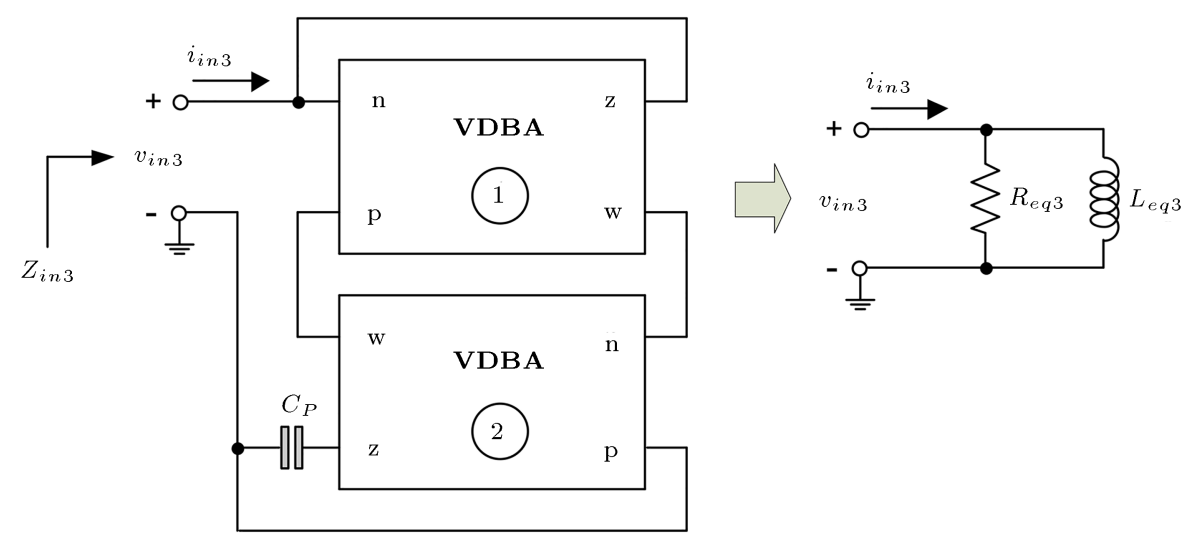

Figure 4. Proposed grounded parallel R-L type lossy inductance simulator.

parallel R-L-type lossy inductors, respectively. In addition, all the proposed circuits do not require any critical element-matching constraints and cancellation choices for the desired realizations. The comparison between the previously similar grounded inductance simulators [2-16] and the proposed VDBA-based simulators in terms of performance is summarized in Table 1.

\subsection{Simulated lossless inductor}

From Eq. (2), the simulated lossless equivalent inductance of Figure 2 is realized as follows: $L_{e q 1}=$
$C_{L} / g_{m 1} g_{m 2}$. It is deduced that the realized equivalent inductance value can be tuned electronically through $g_{m 1}$ and/or $g_{m 2}$ of the corresponding VDBAs. Sensitivity analysis shows that all different sensitivity coefficients of the sim ulated lossless inductor with respect to active and passive elements are found as follows:

$$
S_{C_{L}}^{L_{e q 1}}=1, \quad S_{g_{m 1}}^{L_{e q 1}}=S_{g_{m 2}}^{L_{e q 1}}=-1 .
$$

Recall that sensitivity is the percentage that the dependent variable, $L_{e q 1}$ value in this case, changes relative 
Table 1. Comparison of some exemplary grounded inductance simulators with the proposed VDBA-based simulators.

\begin{tabular}{|c|c|c|c|c|c|c|c|c|c|c|c|}
\hline \multirow[t]{2}{*}{ Reference } & \multirow[t]{2}{*}{$\begin{array}{l}\text { Type of } \\
\text { inductor }\end{array}$} & \multirow[t]{2}{*}{$\begin{array}{l}\text { No. of } \\
\text { active } \\
\text { element }\end{array}$} & \multicolumn{2}{|c|}{$\begin{array}{l}\text { No. of } \\
\text { resistors }\end{array}$} & \multicolumn{2}{|c|}{$\begin{array}{c}\text { No. of } \\
\text { capacitors }\end{array}$} & & \multirow[t]{2}{*}{ 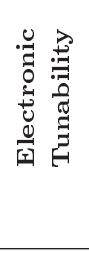 } & \multirow[t]{2}{*}{ 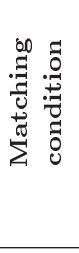 } & \multirow[t]{2}{*}{ 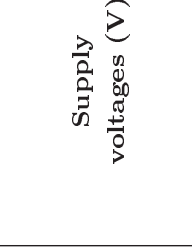 } & \multirow[t]{2}{*}{ 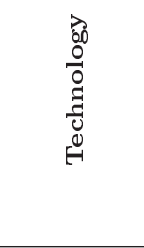 } \\
\hline & & & grounded & floating & grounded & floating & & & & & \\
\hline$[2]$ & Fig. 2 & Lossless & 1 & 1 & 1 & 0 & 1 & No & Yes & $\begin{array}{c} \pm 2.5,-0.604 \\
-0.25\end{array}$ & $\begin{array}{c}\text { TSMC } \\
0.35-\mu \mathrm{m}\end{array}$ \\
\hline$[3]$ & Fig. 1 & Lossless & 1 & 1 & 1 & 0 & 1 & No & Yes & $\pm 1.5,+0.7$ & $\begin{array}{c}\mathrm{TSMC} \\
0.35-\mu \mathrm{m}\end{array}$ \\
\hline \multirow{2}{*}[4]{} & Fig. 2 & Lossless & 3 & 2 & 1 & 1 & 0 & No & No & \pm 5 & AD844 \\
\hline & Fig. 3-4 & & 2 & 1 & 1 & 1 & 0 & No & No & \pm 1.25 & $\begin{array}{c}\mathrm{TSMC} \\
0.25-\mu \mathrm{m}\end{array}$ \\
\hline \multirow[t]{2}{*}[5]{} & Fig. 2(a) & Lossless & 1 & 1 & 1 & 1 & 0 & No & Yes & \pm 1.5 & $\begin{array}{c}\mathrm{TSMC} \\
0.35-\mu \mathrm{m}\end{array}$ \\
\hline & Fig. 2(b)-2(e) & & 1 & 1 & 1 & 1 & 0 & No & No & & \\
\hline$[6]$ & Fig. 1 & Lossless & 2 & 1 & 2 & 0 & 2 & No & Yes & \pm 15 & $\begin{array}{c}\text { MC1458 op } \\
\text { amp }\end{array}$ \\
\hline \multirow[t]{2}{*}[7]{} & Fig. 3(a) & Lossless & 1 & 3 & 0 & 0 & 1 & No & Yes & $\begin{array}{l} \pm 1.65 \\
+0.45\end{array}$ & $\begin{array}{c}\text { AMS } \\
0.35-\mu \mathrm{m}\end{array}$ \\
\hline & Fig. 3(b) & & 1 & 2 & 0 & 0 & 1 & No & Yes & & \\
\hline$[8]$ & Fig. 3 & Lossless & 1 & 0 & 1 & 0 & 1 & Yes & No & \pm 5 & OPA860 \\
\hline \multirow{2}{*}[9]{} & Fig. 2(a) & Series lossy & 1 & 1 & 1 & 1 & 0 & No & No & \pm 2.5 & ALA400 \\
\hline & Fig. 2(b) & Parallel lossy & 1 & 1 & 1 & 1 & 0 & No & No & & \\
\hline$[10]$ & Fig. 2(a) & Parallel lossy & 2 & 0 & 4 & 0 & 1 & No & No & \pm 10 & $\mathrm{AD} 844$ \\
\hline$[11]$ & Fig. 2-5 & Series lossy & 1 & 1 & 1 & 0 & 1 & No & No & \pm 15 & AD 844 \\
\hline \multirow{6}{*}[12]{} & Fig. 2(a) & Lossless & 1 & 1 & 1 & 0 & 1 & No & Yes & $\begin{array}{l} \pm 2.5 \\
+1.44\end{array}$ & $\begin{array}{c}\text { TSMC } \\
0.35-\mu \mathrm{m}\end{array}$ \\
\hline & Fig. 2(b) & Series lossy & 1 & 0 & 2 & 0 & 1 & No & No & & \\
\hline & Fig. 2(c) & Lossless & 1 & 1 & 2 & 0 & 1 & No & Yes & & \\
\hline & Fig. 2(d) & Parallel lossy & 1 & 3 & 0 & 0 & 1 & No & No & & \\
\hline & Fig. 2(e) & Parallel lossy & 1 & 1 & 2 & 0 & 1 & No & No & & \\
\hline & Fig. 2(f) & Series lossy & 1 & 1 & 2 & 0 & 1 & No & No & & \\
\hline \multirow{3}{*}[13]{} & Fig. 3(a) & Series lossy & 1 & 1 & 1 & 1 & 0 & No & No & $\begin{array}{l} \pm 0.75 \\
+0.34\end{array}$ & $\begin{array}{c}\text { IBM } \\
0.13-\mu \mathrm{m}\end{array}$ \\
\hline & Fig. 3(b) & Parallel lossy & 1 & 1 & 1 & 1 & 0 & No & No & & \\
\hline & Fig. 4 & Lossless & 1 & 1 & 1 & 0 & 1 & No & No & & \\
\hline \multirow[t]{3}{*}[14]{} & Fig. 1(a) & Series lossy & 1 & 2 & 0 & 0 & 1 & No & No & \pm 10 & $\begin{array}{c}\text { Bipolar } \\
\text { B101\& } \\
\text { B102 }\end{array}$ \\
\hline & $\begin{array}{c}\text { Fig. } 1(\mathrm{~b})-1(\mathrm{c}) \\
\text { Fig. } 1(\mathrm{e})\end{array}$ & Series lossy & 1 & 1 & 1 & 0 & 1 & No & No & & \\
\hline & Fig. 1(d) & Parallel lossy & 1 & 1 & 2 & 0 & 1 & No & No & & \\
\hline$[15]$ & Fig. 2 & Parallel lossy & 1 & 0 & 2 & 0 & 1 & No & No & \pm 12 & AD844 \\
\hline$[16]$ & Fig. 2 & Parallel lossy & 1 & 0 & 2 & 0 & 0 & No & No & Not reported & $0.5-\mu \mathrm{m}$ \\
\hline \multirow{2}{*}{$\begin{array}{l}\text { Proposed } \\
\text { circuits }\end{array}$} & Fig. 2 & Lossless & 2 & 0 & 0 & 1 & 0 & Yes & No & \pm 0.75 & $\begin{array}{c}\mathrm{TSMC} \\
0.25-\mu \mathrm{m}\end{array}$ \\
\hline & Fig. 3 & Series lossy & & & & & & & & & \\
\hline
\end{tabular}


to the independent variables $\left(g_{m 1}, g_{m 2}\right.$, and $\left.C_{L}\right)$. From Eq. (5), the sensitivity relations are identical except for the sign. Of note, sensitivity of $L_{e q 1}$ with respect to $C_{L}$ is positive. Based on the positive sign, when $C_{L}$ increases, $L_{e q 1}$ also increases. On the other hand, when $g_{m 1}$ increases, $L_{e q 1}$ decreases, which is expected since the sensitivity to $g_{m 1}$ is negative. In other words, the value of $L_{e q 1}$ might vary by very nearly $1 \%$ for every $1 \%$ deviation in either $C_{L}$ or $g_{m i}$ under these conditions. For example, if $g_{m 1}$ or $g_{m 2}$ increases by $1 \%$, then $L_{e q 1}$ becomes a $1 \%$ reduction. Similarly, increase in $C_{L}$ by $1 \%$ causes $1 \%$ increase in $L_{e q 1}$.

It is useful to consider the effect of the VDBA non-idealities on the realized inductor. By taking into account the non-ideal gains $\alpha$ and $\beta$, the equivalent inductance for the lossless simulator circuit shown in Figure 2 is modified as follows:

$$
L_{e q 1}=\frac{C_{L}}{\alpha_{1} \alpha_{2} \beta_{1} \beta_{2} g_{m 1} g_{m 2}},
$$

where $\alpha_{i}$ and $\beta_{i}$ represent the non-ideal gains $\alpha$ and $\beta$ of the related VDBA. This implies that the VDBA non-ideal gains have directly affected the value of $L_{e q 1}$. However, the transconductances $g_{m i}$ of the devices can be fine-tuned to compensate the deviation in the $L_{e q 1}$ value of the proposed simulator circuit.

\subsection{Simulated series $R$-L type lossy inductor}

From Eq. (3), it can be observed that the grounded series R-L impedance with equivalent series resistance $R_{e q 2}=1 / g_{m 1}$ and equivalent series inductance $L_{e q 2}=$ $C_{S} / g_{m 1} g_{m 2}$ is realized from the simulator of Figure 3 . It is worth mentioning that the value of $R_{e q 2}$ value can be adjusted electronically by $g_{m 1}$, while the value of $L_{e q 2}$ may vary without affecting $R_{e q 2}$ by $g_{m 2}$. Moreover, the simulator of Figure 3 can be used as a lossless inductor in the operating frequency region of $\omega>>R_{e q 2} / L_{e q 2}=g_{m 2} / C_{S}$. According to Eq. (3), normalized active and passive sensitivities of $R_{e q 2}$ and $L_{e q 2}$ are derived and obtained as unity in magnitude. The quality factor $\left(Q_{S}\right)$ of this lossy inductor is found as follows:

$$
Q_{S}=\frac{\omega L_{e q 2}}{R_{e q 2}}=\frac{\omega C_{S}}{g_{m 2}} .
$$

If the aforementioned non-ideal gains are taken into consideration, the non-ideal equivalent resistance and inductance of the series lossy inductor in Figure 3 are evaluated as follows:

$$
R_{e q 2}=\frac{1}{\alpha_{1} \beta_{1} \beta_{2} g_{m 1}},
$$

and:

$$
L_{e q 2}=\frac{C_{S}}{\alpha_{1} \alpha_{2} \beta_{1} \beta_{2} g_{m 1} g_{m 2}} .
$$

Note that the small deviation on $R_{e q 2}$ value can sufficiently be minimized by changing $g_{m 1}$, while the error in $L_{e q 2}$ value can be compensated by tuning $g_{m 2}$.

\subsection{Simulated parallel $R-L$ type lossy inductor} From Eq. (4), the simulated equivalent input admittance for the circuit of Figure 4 is obtained as follows:

$$
Y_{i n 3}=\frac{1}{Z_{i n 3}}=\frac{1}{R_{e q 3}}+\frac{1}{s L_{e q 3}}=g_{m 1}+\frac{g_{m 1} g_{m 2}}{s C_{P}} \text {. }
$$

It should be recalled that the circuit of Figure 4 simulates the parallel R-L impedance with the equivalent parallel resistance and inductance values of $R_{e q 3}=$ $1 / g_{m 1}$ and $L_{e q 3}=C_{P} / g_{m 1} g_{m 2}$. One can find that $R_{e q 3}$ is tunable electronically by means of $g_{m 1}$, while $L_{e q 3}$ is adjustable independently through a single transconductance $g_{m 2}$. According to Eq. (10), the proposed circuit of Figure 4 can work as a grounded lossless inductor for $\omega<<R_{e q 3} / L_{e q 3}=g_{m 2} / C_{P}$. Different rates of sensitivity of the simulated $R_{e q 3}$ and $L_{e q 3}$ to active and passive components are also analyzed and it is found that the resulting sensitivity values are all no more than unity in magnitude. For this parallel inductor, the quality factor $\left(Q_{P}\right)$ can be given as follows:

$$
Q_{P}=\frac{R_{e q 3}}{\omega L_{e q 3}}=\frac{g_{m 2}}{\omega C_{p}} .
$$

Taking into account the non-ideal gains $\alpha$ and $\beta$ for the circuit in Figure 4, one can respectively obtain the modified $R_{e q 3}$ and $L_{e q 3}$ as follows:

$$
R_{e q 3}=\frac{1}{\alpha_{1} \beta_{1} g_{m 1}}
$$

and:

$$
L_{e q 3}=\frac{C_{P}}{\alpha_{1} \alpha_{2} \beta_{1} \beta_{2} g_{m 1} g_{m 2}} .
$$

\section{Simulations, discussions, and applications}

The validity of all the proposed grounded inductance simulators in Figures 2-4 has been evaluated using PSPICE simulation program. To achieve simulations, the VDBA is derived from the schematic CMOS implementation illustrated in Figure 5 with symmetrical supply voltages of $\pm 0.75 \mathrm{~V}$ and biasing currents $I_{A}$ of 50 $\mu \mathrm{A}$ [23]. CMOS transistors in Figure 5 are simulated with the real process parameters of $0.25-\mu \mathrm{m}$ CMOS technology from TSMC. Transistor sizes $(W / L)$ are given in Table 2. Based on the internal structure of Figure 5, let's assume that $M_{1}-M_{2}$ of the source couple are well matched and the current mirror $M_{3}-M_{4}$ has unity current transfer gain. Therefore, the value of transconductance $g_{m}$ of the VDBA can be determined by [23]:

$$
g_{m}=\sqrt{K I_{B}}
$$

where $I_{B}$ is the external bias current of the VDBA, 


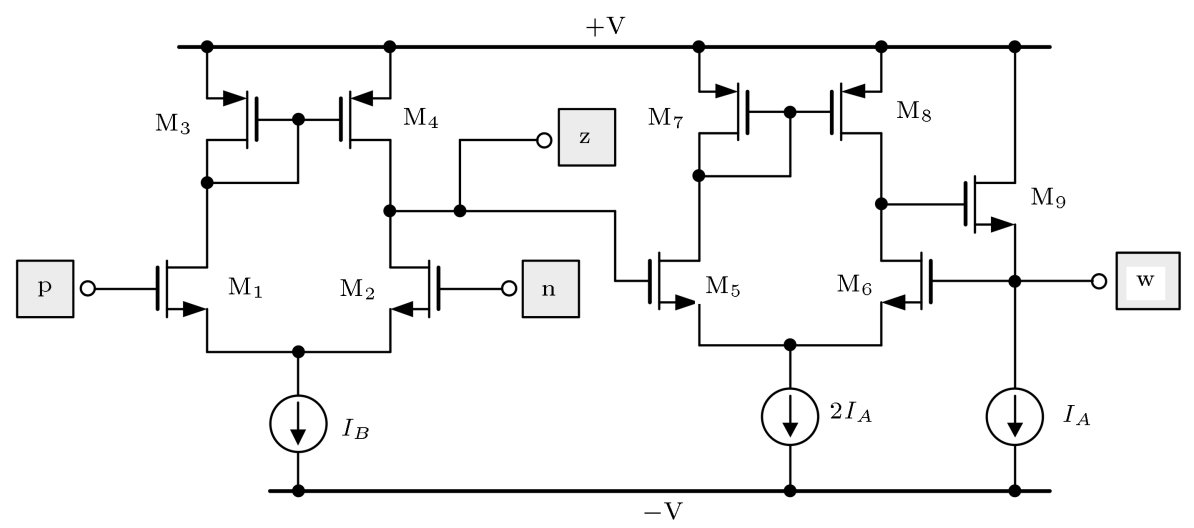

Figure 5. CMOS realization for the VDBA adopted from [23].

Table 2. Dimensions of the CMOS transistors of the VDBA circuit in Figure 5.

\begin{tabular}{cc}
\hline Transistors & $\boldsymbol{W}(\boldsymbol{\mu m}) / \boldsymbol{L}(\boldsymbol{\mu m})$ \\
\hline $\mathrm{M}_{1}-\mathrm{M}_{2}, \mathrm{M}_{5}-\mathrm{M}_{6}$ & $25 / 0.25$ \\
$\mathrm{M}_{3}, \mathrm{M}_{7}$ & $32 / 0.25$ \\
$\mathrm{M}_{4}, \mathrm{M}_{8}$ & $35 / 0.25$ \\
$\mathrm{M}_{9}$ & $25 / 0.25$ \\
\hline
\end{tabular}

and $K=\mu C_{o x} W / L$ is the transconductance coefficient, in which $\mu$ is carrier mobility, $C_{o x}$ is the gate oxide capacitance per unit area, and $W$ and $L$ are the channel width and length of $M_{1}$ and $M_{2}$ devices, respectively. Eq. (14) clearly indicates that the $g_{m}$ value can be adjusted electronically with adjustment of $I_{B}$.

\subsection{Proposed lossless inductor in Figure 2}

The proposed grounded lossless inductance simulator circuit shown in Figure 2 is simulated with the following component values: $g_{m}=g_{m 1}=g_{m 2} \cong 1.50 \mathrm{~mA} / \mathrm{V}$ and $C_{L}=50 \mathrm{pF}$. For a given transconductance value, the corresponding bias currents calculated using Eq. (14) are equal to $I_{B}=I_{B 1}=I_{B 2}=90 \mu \mathrm{A}$. Based on Eq. (2), the simulated equivalent inductance is obtained as: $L_{e q 1}=22.22 \mu \mathrm{H}$. Figure 6 shows the

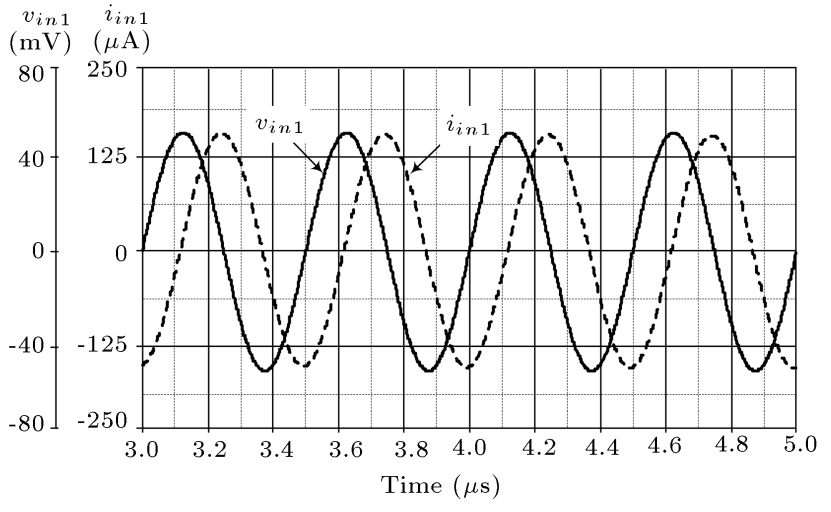

Figure 6. Simulated transient waveforms of $v_{i n 1}$ and $i_{i n 1}$ for the proposed lossless inductor of Figure 2. simulated transient waveforms of the voltage $v_{i n 1}$ and the current $i_{i n 1}$ through the proposed lossless inductor of Figure 2, for a sinusoidal input signal with $50 \mathrm{mV}$ peak value at $f=2 \mathrm{MHz}$. Based on the results of Figure 6 , the phase difference between $v_{i n 1}$ and $i_{i n 1}$ is equal to $83.5^{\circ}$ lagging, where its ideal value is $90^{\circ}$ lagging. Total power consumption of the circuit is measured as $0.653 \mathrm{~mW}$. On the other hand, the ideal and simulated frequency characteristics of the input impedance $Z_{i n 1}$ of the proposed lossless inductor with the same component setting are shown in Figure 7 . It is shown that for the acceptable phase error less than $8 \%$, the operating frequency range of the simulator is approximately $100 \mathrm{kHz}$ up to $70 \mathrm{MHz}$. Furthermore, to illustrate the electronic tuning property of the synthetic inductor, the impedance magnitude frequency characteristics with variation in the equivalent inductance value by tuning $I_{B}$ as: $50 \mu \mathrm{A}$ (for $L_{e q 1}=40 \mu \mathrm{H}$ ), 150 $\mu \mathrm{A}\left(\right.$ for $\left.L_{e q 1}=13.3 \mu \mathrm{H}\right)$, and $300 \mu \mathrm{A}$ (for $L_{e q 1}=6.7$ $\mu \mathrm{H})$ are given in Figure 8 . The resulting characteristics prove that the variation of $L_{e q 1}$ can be performed electronically by changing the control current $I_{B}$.

The functionality of the simulated lossless inductor of Figure 2 has been verified by using it in the realization of a tunable voltage-mode bandpass (BP)

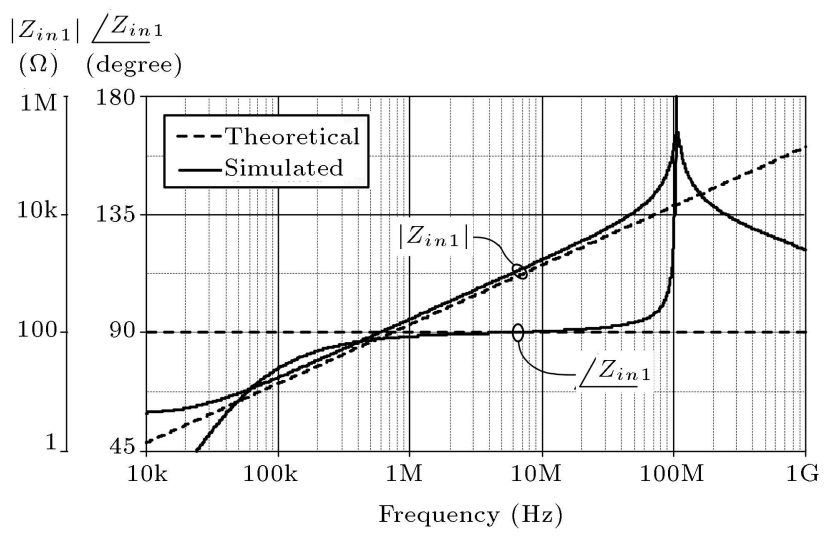

Figure 7. Theoretical and simulated frequency responses of $Z_{i n 1}$ for the proposed lossless inductor of Figure 2. 


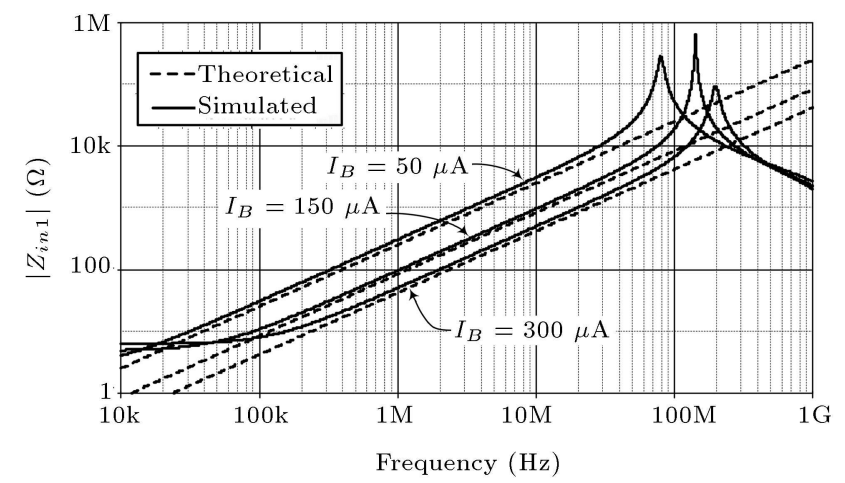

Figure 8. Impedance magnitude-frequency responses of Figure 2 for three different values of $I_{B}$.

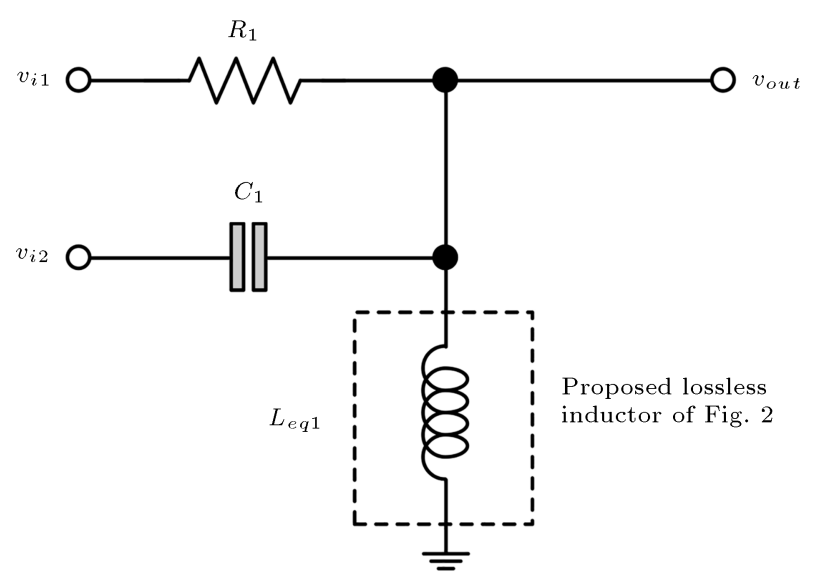

Figure 9. Tunable voltage-mode BP and HP filters constructed with the proposed lossless inductor in Figure 2.

and highpass (HP) filter shown in Figure 9 [4]. The voltage transfer functions are given as follows:

$$
B P=\frac{V_{\text {out }}(s)}{V_{i 1}(s)}=\frac{\left(\frac{\omega_{o}}{Q}\right) s}{s^{2}+\left(\frac{\omega_{o}}{Q}\right) s+\omega_{o}^{2}},
$$

and:

$$
H P=\frac{V_{\text {out }}(s)}{V_{i 2}(s)}=\frac{s^{2}}{s^{2}+\left(\frac{\omega_{o}}{Q}\right) s+\omega_{o}^{2}},
$$

where $\omega_{o}$ and $Q$ are the natural angular frequency and the quality factor of the filter, respectively. From Eqs. (15) and (16), the characteristics $\omega_{0}$ and $Q$ of the $\mathrm{BP}$ and $\mathrm{HP}$ voltage responses are equal to:

$$
\omega_{o}=2 \pi f_{o}=\frac{1}{\sqrt{L_{e q 1} C_{1}}},
$$

and:

$$
Q=R_{1} \sqrt{\frac{C_{1}}{L_{e q 1}}} .
$$

As an example, the values of the passive elements used in the realized filter are taken as $R_{1}=200 \Omega$ and

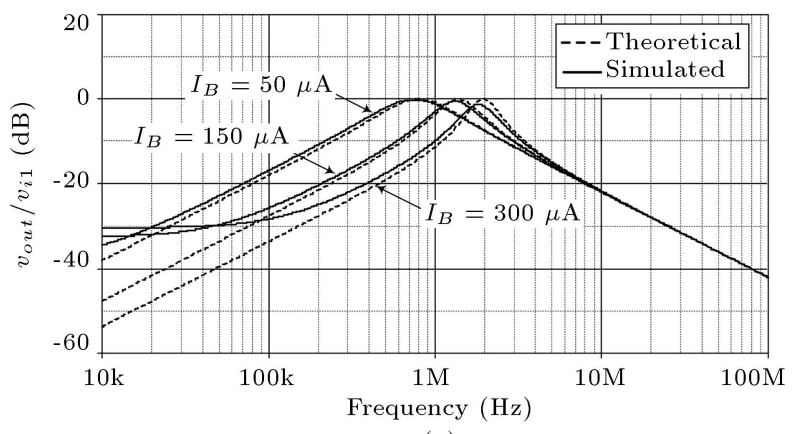

(a)

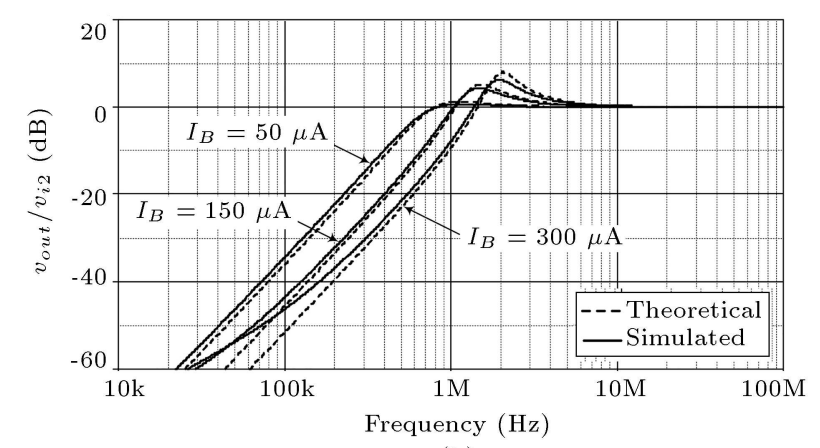

(b)

Figure 10. Gain-frequency responses of the filter realization in Figure 9 for three different values of $I_{B}$.

$C_{1}=1 \mathrm{nF}$. The gain-frequency responses of the BP and HP filters obtained from theoretical experiments and simulation with variation in $I_{B}$ are depicted in Figure 10. In the case of IB $=50 \mu \mathrm{A}, 150 \mu \mathrm{A}$, and $300 \mu \mathrm{A}$, the filter parameters are calculated as $f_{o}=0.8$ $\mathrm{MHz}, 1.4 \mathrm{MHz}$ and $2 \mathrm{MHz}$, and $Q=1,1.73$, and 2.45, respectively.

\subsection{Proposed series lossy inductor in Figure 3} For the simulation of $R_{e q 2}=666 \Omega$ and $L_{e q 2}=22.21$ $\mu \mathrm{H}$ by using the grounded series lossy inductor in Figure 3, the designed active and passive components taken from Eqs. (3) and (14) include $I_{B}=I_{B 1}=I_{B 2}=$ $90 \mu \mathrm{A}$ and $C_{S}=50 \mathrm{pF}$. Figure 11 shows the simulated

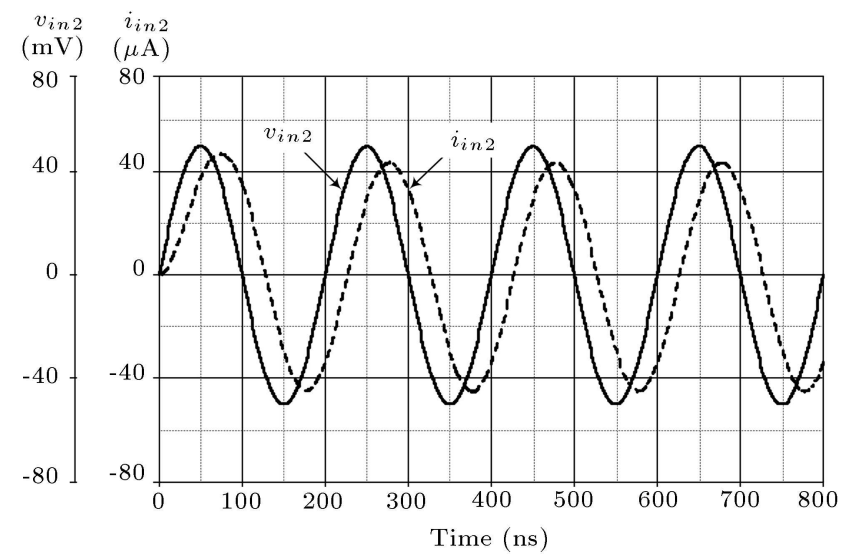

Figure 11. Simulated transient waveforms of $v_{i n 2}$ and $i_{\text {in } 2}$ for the proposed series lossy inductor of Figure 3 . 


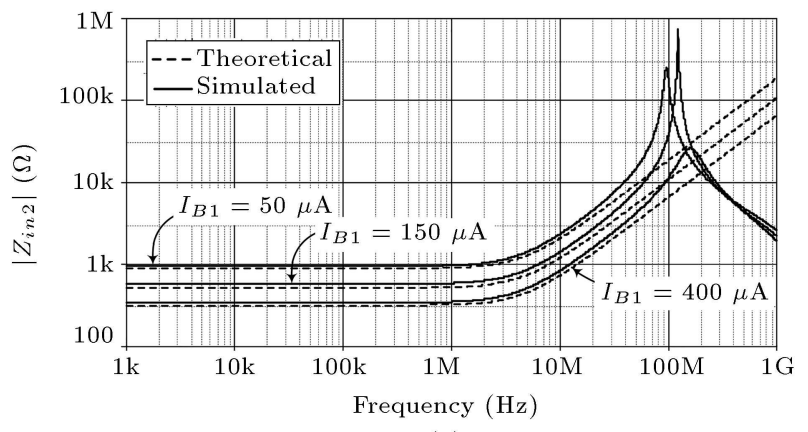

(a)

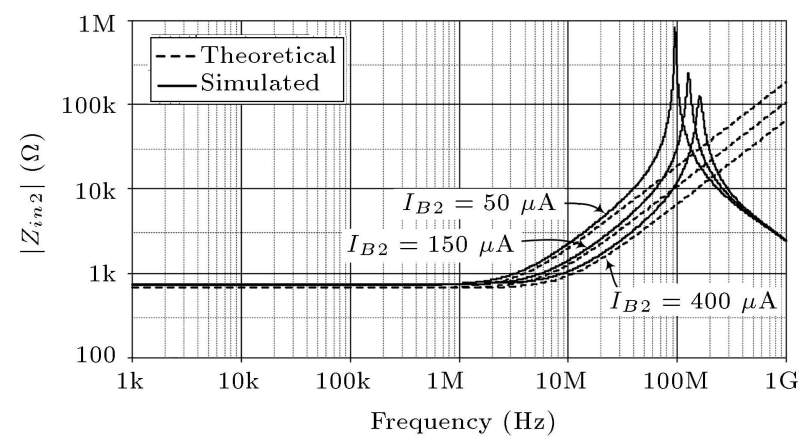

(b)

Figure 12. Magnitude-frequency responses for $Z_{\text {in2 } 2}$ of Figure 3: (a) with $I_{B 1}$ tuning and (b) with $I_{B 2}$ tuning.

input waveforms through the simulator when applying a $5-\mathrm{MHz}$ sinusoidal signal with $50 \mathrm{mV}$ peak. It appears that the phase angle of the current $i_{i n 2}$ delays that of the voltage $v_{i n 2}$ by $48^{\circ}$, where the theoretically predicted value is equal to $46^{\circ}$. Figure 12 (a) illustrates the magnitude-frequency characteristics of $Z_{i n 2}$ for $I_{B 2}=$ $90 \mu \mathrm{A}$ and various values of $I_{B 1}$ (i.e., $50 \mu \mathrm{A}, 150 \mu \mathrm{A}$ and $400 \mu \mathrm{A}$ ). According to Eq. (3), the corresponding $R_{e q 2}$ and $L_{e q 2}$ are obtained as: $\left(R_{e q 2}=894 \Omega\right.$ and $\left.L_{e q 2}=30 \mu \mathrm{H}\right),\left(R_{e q 2}=516 \Omega\right.$ and $\left.L_{e q 2}=17 \mu \mathrm{H}\right)$, and $\left(R_{e q 2}=316 \Omega\right.$ and $\left.L_{e q 2}=10.5 \mu \mathrm{H}\right)$, respectively. On the other hand, to show the independent electronic tuning of the $L_{e q 2}$ value, the circuit component values are chosen as follows: $C_{S}=50 \mathrm{pF}, I_{B 1}=90 \mu \mathrm{A}$, and $I_{B 2}$ changing from $50 \mu \mathrm{A}, 150 \mu \mathrm{A}$ to $400 \mu \mathrm{A}$, leading to $R_{e q 2}=666 \Omega$ and $L_{e q 2}$ varying from $30 \mu \mathrm{H}, 17 \mu \mathrm{H}$ to $10.5 \mu \mathrm{H}$. Both of the theoretical and simulated results are given in Figure 12(b). As it is readily seen from these characteristics, the $R_{e q 2}$ value is electronically tunable through $I_{B 1}\left(g_{m 1}\right)$, while the $L_{e q 2}$ value is orthogonally adjustable through $I_{B 2}\left(g_{m 2}\right)$.

To further evaluate the behavior of the proposed series lossy inductor in Figure 3 , it is tested on an active RLC current-mode lowpass (LP) filter given in Figure 13. The active and passive component values used for Figure 13 are as follows: $I_{B 1}=90 \mu \mathrm{A}$, $C_{S}=50 \mathrm{pF}$, and $C_{2}=47 \mathrm{pF}$. Figure 14 shows the simulated frequency responses of the filter with three different values of $I_{B 2}$. As a result, the achieved cutoff frequency and the quality factor of this filter are

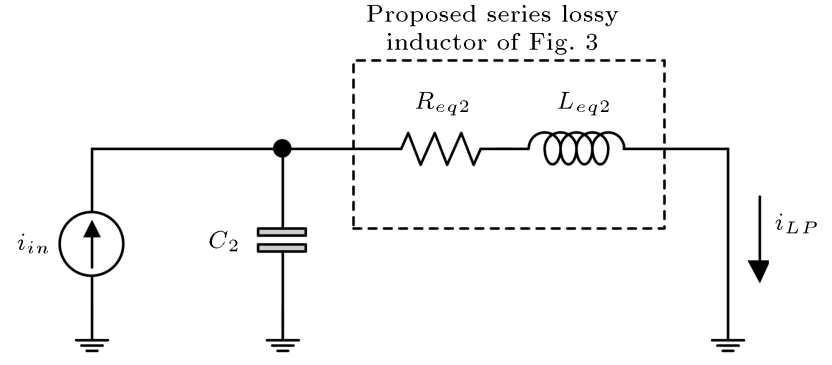

Figure 13. Tunable current-mode LP filter realization using the proposed series lossy inductor of Figure 3.

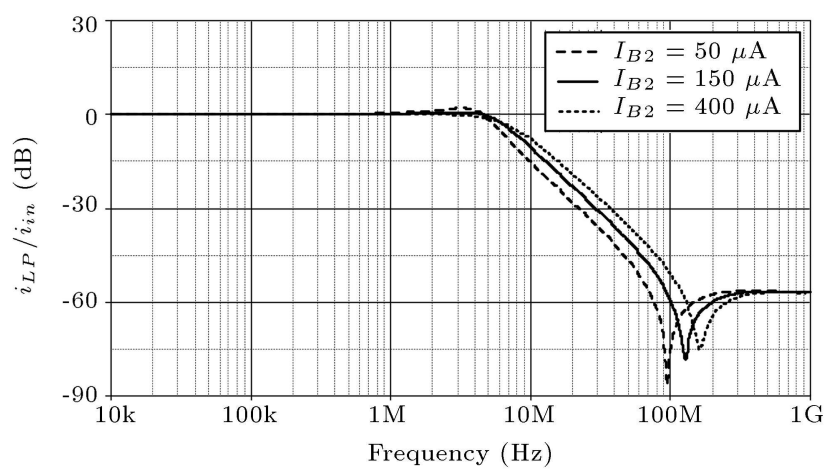

Figure 14. Simulated frequency responses of LP filter in Figure 13.

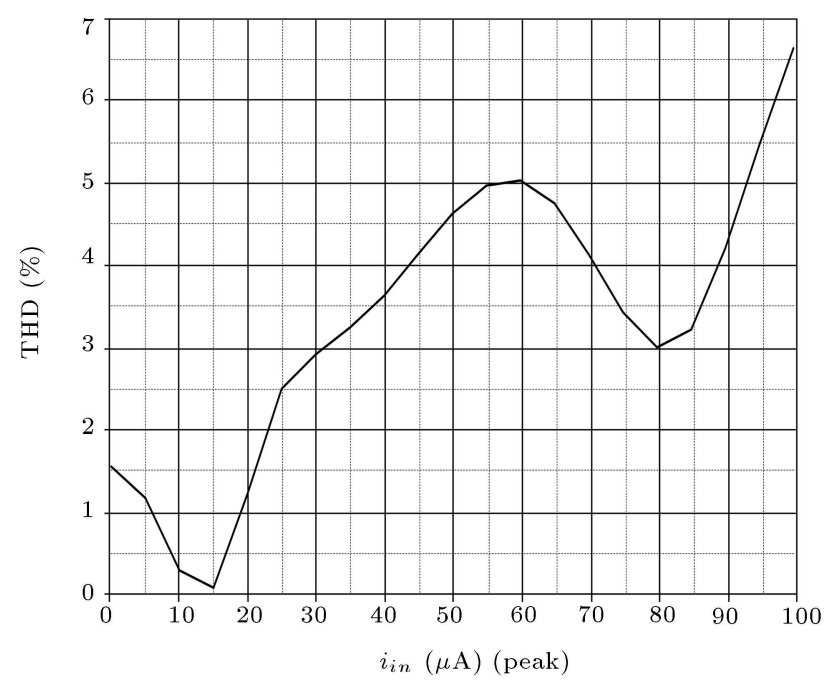

Figure 15. THD variations of LP filter in Figure 13 against applied input signal amplitude.

about: $f_{o}=1 / 2 \pi\left(L_{e q 2} C_{2}\right)^{1 / 2} \cong 4.25 \mathrm{MHz}, 5.60 \mathrm{MHz}$, $7.15 \mathrm{MHz}$ and $Q=\left(1 / R_{e q 2}\right)\left(L_{e q 2} / C_{2}\right)^{1 / 2} \cong 1.2,0.9$, and 0.7 , respectively. In addition, the Total Harmonic Distortion (THD) variations of $i_{L P}$ in Figure 13 against an applied sinusoidal input current amplitude at $f_{o}=$ 4.25 $\mathrm{MHz}$ are given in Figure 15.

\subsection{Proposed Parallel Lossy Inductor in Figure 4}

In the case of the proposed grounded parallel R-L type lossy inductor in Figure 4, the component values are 


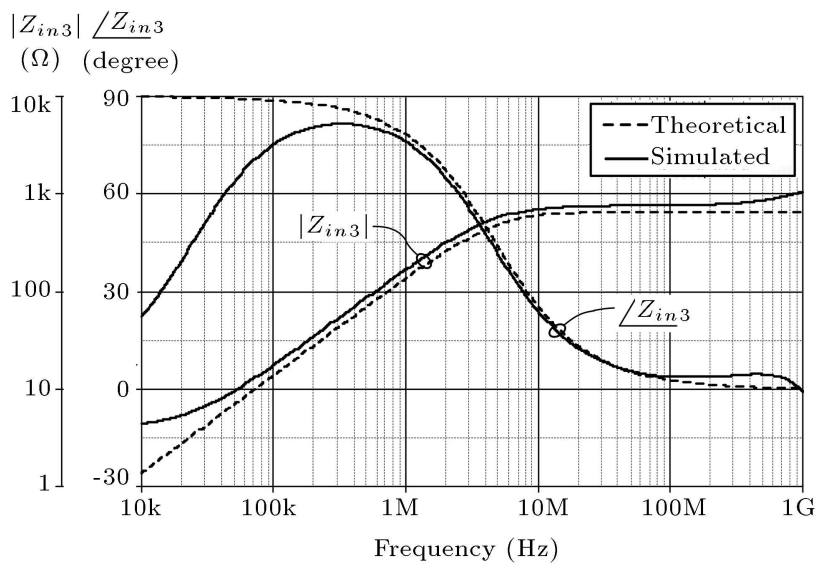

Figure 16. Theoretical and simulated frequency responses of $Z_{i n 3}$ for the proposed parallel lossy inductor of Figure 4.

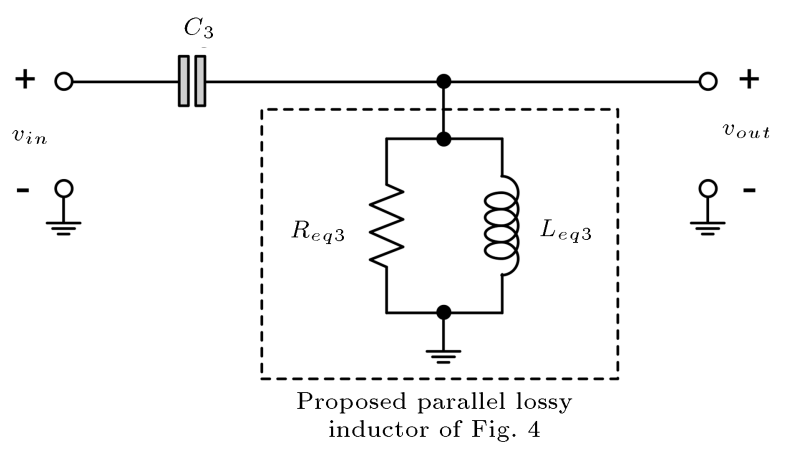

Figure 17. Tunable voltage-mode HP filter realization using the proposed parallel lossy inductor of Figure 4.

chosen as $I_{B 1}=I_{B 2}=90 \mu \mathrm{A}$ and $\mathrm{CP}=50 \mathrm{pF}$ so as to obtain the ideal values of $R_{e q 2}=666 \Omega$ and $L_{e q 2}=22.21 \mu \mathrm{H}$. The magnitude and phase characteristics of $Z_{i n 3}$ relative to frequency are given in Figure 16. The practical usability of the proposed parallel inductor is demonstrated on the voltage-mode HP filter shown in Figure 17. To design a filter with $f_{o}=\omega_{o} / 2 \pi\left(L_{e q 3} C_{3}\right)^{1 / 2} \cong 4.67 \mathrm{MHz}, 6.14 \mathrm{MHz}, 7.85$ $\mathrm{MHz}$, and $Q=R_{e q 3}\left(C_{3} / L_{e q 3}\right)^{1 / 2} \cong 0.76,1,1.28$, it is realized by taking $C_{3}=40 \mathrm{pF}, I_{B 1}=90 \mu \mathrm{A}$, and $I_{B 2}=50 \mu \mathrm{A}, 150 \mu \mathrm{A}$, and $400 \mu \mathrm{A}$ and the following values are respectively obtained as a result: $R_{e q 3}=666 \Omega$ and $L_{e q 3}=30 \mu \mathrm{H}, 17 \mu \mathrm{H}$, and $10.5 \mu \mathrm{H}$. The simulated frequency responses of the realized HP filter upon tuning $I_{B 2}$ value are given in Figure 18.

\section{Conclusions}

Three configurations for simulating grounded lossless and lossy inductors were presented in this study. All the circuits employed two VDBAs and one grounded capacitor. The realized equivalent element values were tuned electronically by adjusting the bias currents of the VDBAs. Moreover, all the proposed inductance

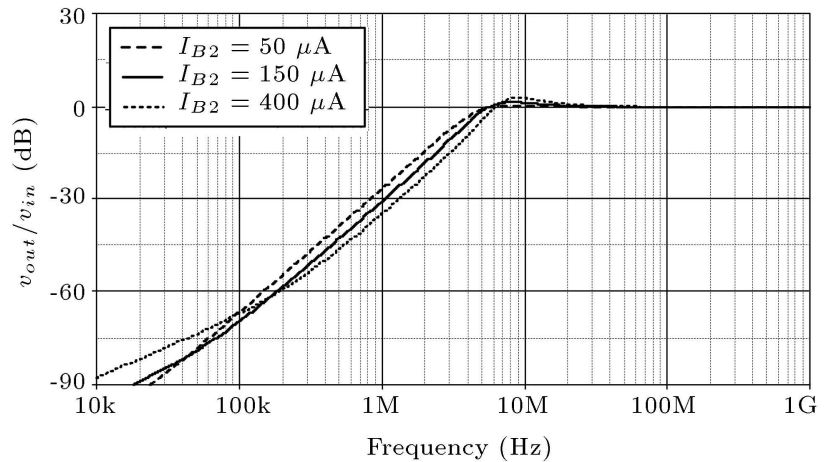

Figure 18. Simulated frequency responses of HP filter in Figure 17 for different $I_{B 2}$ values.

simulator circuits did not need any critical constraints of component matching. The effects of VDBA nonideal gains were examined. Several simulation results based on TSMC $0.25-\mu \mathrm{m}$ CMOS technology were performed to verify the theoretical analysis. To demonstrate the viability of the proposed inductors, some examples of applications were also included.

\section{Acknowledgments}

This work was supported by King Mongkut's Institute of Technology Ladkrabang Research Fund [grant number KREF116001]. The authors are also immensely grateful to Mr. Natchanai Roongmuanpha for assistance with the circuit simulation who moderated this paper and improved the manuscript significantly.

\section{References}

1. Moezzi, M. and Bakhtiar, M.S. "A tunable high-Q active inductor with a feed forward noise reduction path", Scientia Iranica, Trans. D: Comp. Science \& Eng. Electr. Eng., 21(3), pp. 945-952 (2014).

2. Yuce, E., Minaei S., and Cicekoglu, O. "A novel grounded inductor realization using a minimum number of active and passive components", ETRI Journal, 27(4), pp. 427-432 (2005).

3. Yuce, E. "Inductor implementation using a canonical number of active and passive elements", Int. J. Electron., 94(4), pp. 317-326 (2007).

4. Yuce, E. and Minaei, S. "On the realization of simulated inductors with reduced parasitic impedance effects", Circuits Syst. Signal Process., 28(3), pp. 451465 (2009).

5. Kacar, F. "New lossless inductance simulators realization using a minimum active and passive components", Microelectron. J., 41(2-3), pp. 109-113 (2010).

6. Maundy, B. and Gift, S.J.G. "Active grounded inductor circuit", Int. J. Electron., 98(5), pp. 555-567 (2011).

7. Myderrizi, I., Minaei, S., and Yuce, E. "DXCCIIbased grounded inductance simulators and filter ap- 
plications", Microelectron. J., 42(9), pp. 1074-1081 (2011).

8. Yesil, A., Kacar, F., and Gurkan, K. "Lossless grounded inductance simulators employing single VDBA and its experimental band-pass filter application", Int. J. Electron. Commun. (AEU), 68(2), pp. 143-150 (2014).

9. Metin, B. "Canonical inductor simulators with grounded capacitors using DCCII", Int. J. Electron., 99(7), pp. 1027-1035 (2012).

10. Cam, U., Kacar, F., Cicekoglu, O., et al. "Novel two OTRA-based grounded immittance simulator topologies", Analog Integr. Circ. Sig. Process., 39(2), pp. 169-175 (2004).

11. Yuce, E. "Novel lossless and lossy grounded inductor simulators consisting of a canonical number of components", Analog Integr. Circ. Sig. Process., 59(1), pp. 77-82 (2009).

12. Metin, B. "Supplementary inductance simulator topologies employing single DXCCII", Radioengineering, 20(3), pp. 614-618 (2011).

13. Alpaslan, H. and Yuce, E. "Inverting CFOA based lossless and lossy grounded inductor simulators", Circuits Syst. Signal Process., 34(10), pp. 3081-3100 (2015).

14. Kuntman, H., Gulsoy, M., and Cicekoglu, O. "Actively simulated grounded lossy inductors using third generation current conveyors", Microelectron. J., 31(4), pp. 245-250 (2000).

15. Pathak, J.K., Singh A.K., and Senani, R. "New canonical lossy inductor using a single CDBA and its application", Int. J. Electron., 103(1), pp. 1-13 (2016).

16. Senani, R., Singh, A.K., Gupta, A., and Bhaskar, D.R. "Simple simulated inductor, low-pass/band-pass filter and sinusoidal oscillator using OTRA", Circuits and Systems, 7(3), pp. 83-99 (2016).

17. Jaikla, W. and Lahiri, A. "Current feedback opamp based linear voltage-controlled oscillator using analog multipliers and minimum passive components", Scientia Iranica, Trans. D: Comp. Science \& Eng. Electr. Eng., 23(3), pp. 1294-1300 (2016).

18. Bhushan, M. and Newcomb, R.W. "Grounding of capacitors in integrated circuits", Electron. Lett., 3, pp. 148-149 (1967).

19. Sun, Y. "Design of high frequency integrated analogue filters", IET Circuits, Devices and Systems Series, 14, pp. 1-35 (2002).
20. Kacar, F., Yesil, A., and Noori, A. "New CMOS realization of voltage differencing buffered amplifier and its biquad filter applications", Radioengineering, 21(1), pp. 333-339 (2012).

21. Sotner, R., Jerabek, J., and Herencsar, N. "Voltage differencing buffered/inverted amplifiers and their applications for signal generation", Radioengineering, 22(2), pp. 490-504 (2013).

22. Tangsrirat, W., Surakampontron, W., and Fujii, N. "Realization of leapfrog filters using current differential buffered amplifiers", IEICE Trans. Fundamental., E86-A(2), pp. 318-326 (2003).

23. Tangsrirat, W. "Actively floating lossy inductance simulators using voltage differencing buffered amplifiers", IETE Journal of Research, 65(4), pp. 446-459 (2019). DOI: $10.1080 / 03772063.2018 .1433082$

\section{Biographies}

Worapong Tangsrirat received the BIndTech degree (Honors) in Electronics Engineering as well as MEng and DEng degrees in Electrical Engineering, all from the Faculty of Engineering, King Mongkut's Institute of Technology Ladkrabang (KMITL), Bangkok, Thailand in 1991, 1997, and 2003, respectively. Since 1995, he has been a faculty member at KMITL, where he is currently a Full Professor at the Department of Instrumentation and Control Engineering. His research interests are mainly in analog signal processing and integrated circuits, current-mode circuits, and active filter and oscillator design.

Wanlop Surakampontorn received his BEng and MEng degrees in Electrical Engineering from King Mongkut's Institute of Technology Ladkrabang (KMITL), Bangkok, Thailand, in 1976 and 1978, respectively, and the $\mathrm{PhD}$ in Electronics from the University of Kent at Canterbury, Kent, UK in 1983. Now, he has been a member of the College of Advanced Manufacturing Innovation, KMITL, where he is currently a Senior Professor of Electronic Engineering. His research interests are in the areas of analog and digital integrated circuit designs, real-time application of PC computers and microprocessors, digital signal processing, electronic instrumentation, and VLSI signal processing. He is a senior member of the IEEE and is a member of the IEICE. 